\title{
Classical Elephant Trunk-old fashioned thus obsolete?
}

\author{
Wim J. Morshuis \\ Department of Cardiothoracic Surgery, Radboud University Medical Center, Nijmegen, The Netherlands \\ Correspondence to: Wim J. Morshuis. Department of Cardiothoracic Surgery, Radboud University Medical Center, Nijmegen, The Netherlands. \\ Email: wim.morshuis@radboudumc.nl.
}

Received: 03 January 2021; Accepted: 12 October 2021; Published online: 09 November 2021.

doi: 10.21037 /jovs-20-7

View this article at: https://dx.doi.org/10.21037/jovs-20-7

To address aortic arch aneurysms by minimal invasive stent grafting is appealing, however, stroke and mortality rates are of still of major concern, although these adverse events mostly address patients not suitable for open arch surgery. With regards to the reported results (1), it is important to realize that these are the best possible results and are reported from high volume centers, whereas attempts of small centers are probably never known especially the disappointing ones. We must also realize that that minimal invasive techniques have a price as learning curves are steep and while learning patients may die or may be damaged (2). Open surgery of the arch is certainly not without complications as well but more teachable when maintaining a strict protocol. A protocol based on protection of the brain and the body during the period of deep hypothermic circulatory arrest. Antegrade selective cerebral perfusion is the most logical way to protect the brain, always bilateral, as the collateral circulation of the brain may show incomplete variations rendering unilateral brain perfusion alone unsafe or moderately unsafe in up to $30 \%$ of the cases (3).

In 2017, we reviewed the results of elective arch surgery with regard to neurological outcome (3). Mean age was 63 years, almost $18 \%$ had a reoperation. Total arch replacement, including classical elephant trunk (ET), was performed in 228 patients. Hospital mortality was low $(5.3 \%)$; permanent neurological damage was seen in $5.3 \%$. Marfan syndrome, femoral artery cannulation, reoperation and the use of the frozen elephant trunk (FET) emerged as risk factors for stroke, whereas older age and the use of bilateral brain perfusion seemed to be protective.

In total arch replacement with the use of the classical ET, patients are cooled down to a central body temperature (bladder or rectal) of at least 25 degrees. At circulatory arrest, first the distal anastomosis is made using side bites on the prosthesis, or using a so called Sienna prosthesis with a side cuff in case of dilatation at the suture side. In between this distal anastomosis and the subclavian artery reimplantation, we reperfuse the body for 10 minutes, as we have learned it to be beneficial in operation for CTEPH using intermittent circulatory arrest. This is important because the reimplantation of the left subclavian artery can be most cumbersome and time consuming, especially with large aneurysms with remote position of the artery and in case of dissected or calcified arterial wall. After attaching the subclavian artery, body perfusion is restarted (Figure 1), then dealing first with the ascending pathology so that the heart can start beating. Only thereafter the remaining arch vessels are reconnected. Nowadays we tend to make our distal anastomosis in zone 2, with low offspring of the side branches (Figure 2), to allow a hybrid solution of a distal arch problem but also to avoid damaging the recurrent nerve, which can be an invalidating complication, especially in young patients. We have seen that the extension of the total aortic ach replacement with a conventional ET did not influence neurological outcome (3) and allows for completion by TEVAR or open thoraco-or thoracoabdominal extension (Figure 3). When we reviewed the results of the conventional ET 4 years ago in a relatively complex group of almost 250 patients (4), the mortality rate was $8 \%$, with low complication rate and no cases of paraplegia, satisfactory, however, with 125 patients planned for second stage, 8 patients died waiting as in a patient with a two months interval and only 2 weeks before planned TEVAR (Figure 4). In an effort to attack this interval mortality, and thus changing a two stage procedure into a one stage procedure, the Frozen ET was introduced, with initial high complication rates (5-7) such as paraplegia, something we have not seen in our series of the conventional ET, maybe the price of 


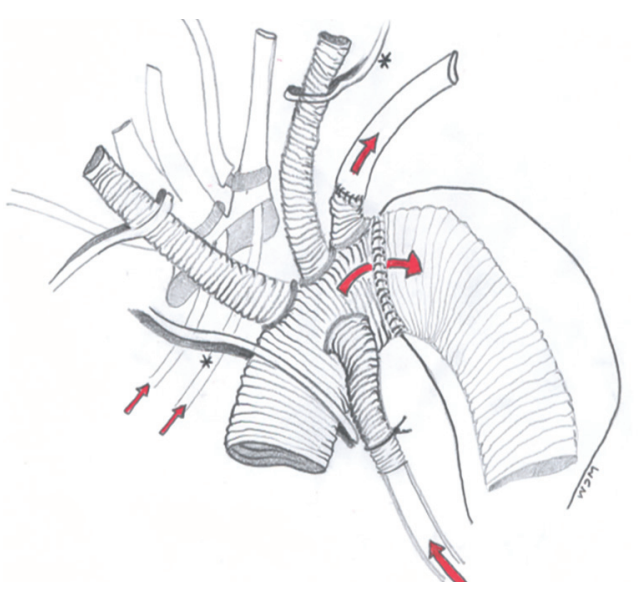

Figure 1 After attaching the subclavian artery, body perfusion is restarted, then finishing first the ascending pathology as to diminish cardiac arrest time.

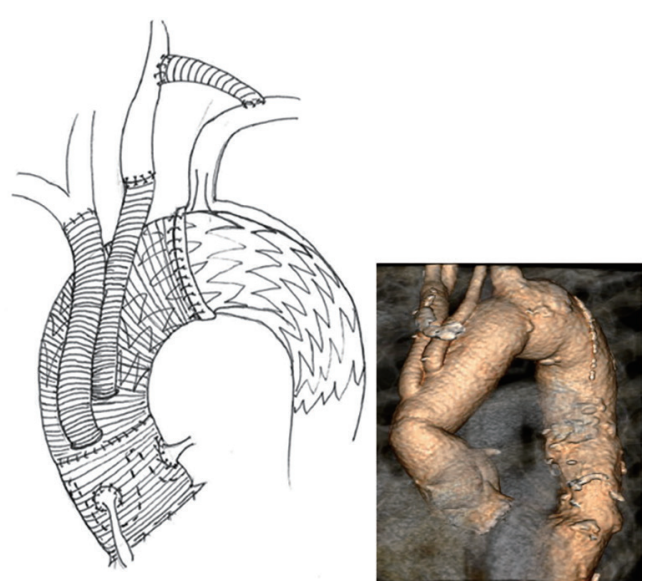

Figure 2 Distal anastomosis in zone 2 with low offspring of the side branches.

innovation.

Adjustments were made. The length of the frozen was reduced to only $10 \mathrm{~cm}$, thereby also decreasing the suitable cases for a true one stage procedure and also spinal fluid drainage in elective cases was now added. The E-Vita concept was gradually replaced by a new 4-branched prosthesis, with the advantages of shorter body and cardiac ischemic times (8).

The results of these changes of should be read from recent studies of two of the largest European aortic centers, Bologna and Hannover, comparing also two cohorts of
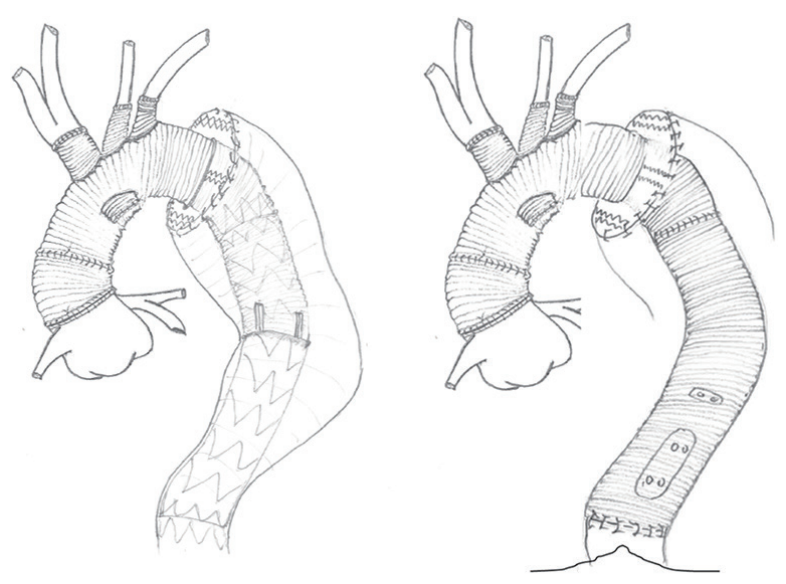

Figure 3 Second stage classical ET completion by TEVAR or open thoraco-or thoracoabdominal extension. ET, elephant trunk. TEVAR, thoracic endovascular aortic repair.

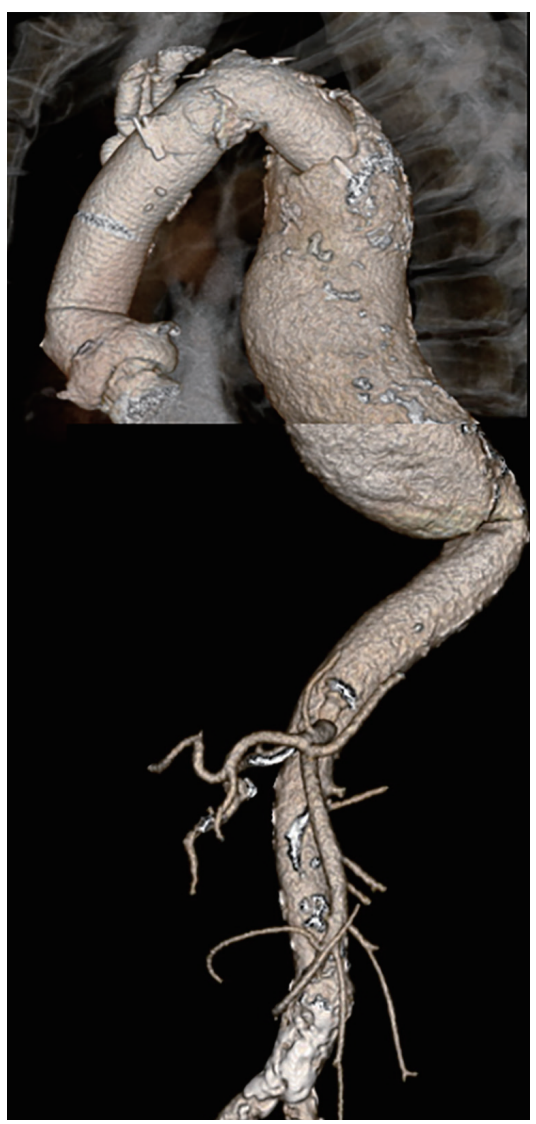

Figure 4 Interval mortality. Patient died 2 months after aortic arch operation of descending aorta rupture only 2 weeks before planned TEVAR. TEVAR, thoracic endovascular aortic repair. 

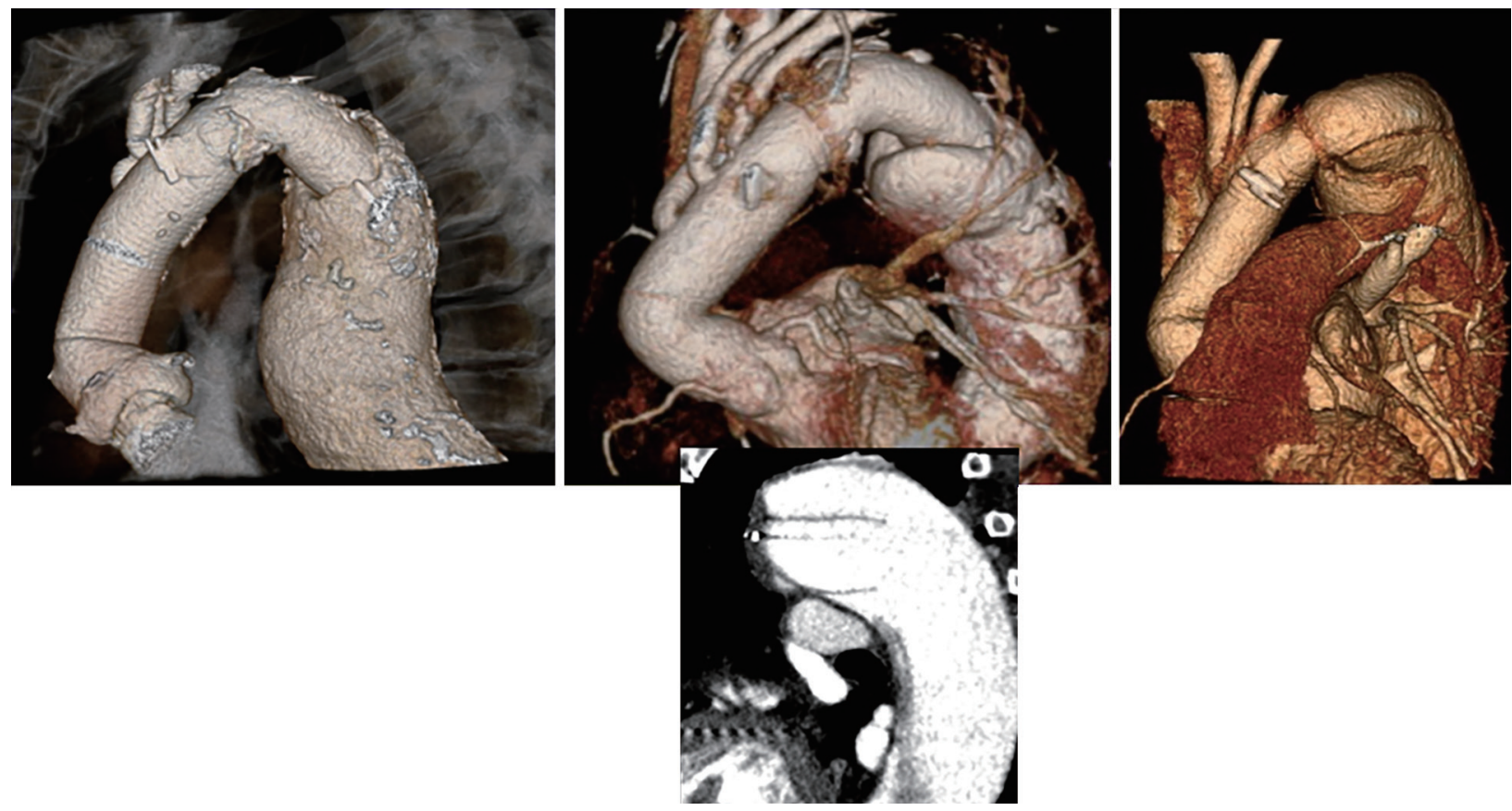

Figure 5 When pressurized, the classical elephant trunk will stretch out facilitating TEVAR positioning. TEVAR, thoracic endovascular aortic repair.

time. In the latest era mostly predominantly using the Thoraflex prothesis with the separate graft technique. And indeed, in Hannover (9), for chronic dissections, mortality has gone down to zero, with only cases of paraparesis and no paraplegia, however, with more than doubling recurrent nerve palsy and still with significant stroke rate (7.3\%). For degenerative aneurysms the results are more worrisome with permanent dialysis and paraplegia in $7.3 \%$, stroke in $17 \%$ and a high mortality, especially when compared to their own results of the classical ET which they described in 2015 (10), even including acute type A dissection.

In another study of Hannover, the question arises whether the frozen is superior for the completion of the second stage operation (11). In their conclusion they state that mortality is lower for the FET group. The question is if this is an honest conclusion. First, there seems to be a bias in patient selection. The FET group has no stroke after the first operation where we have seen this to be as high as $14 \%$ as we may have read from their total experience (10), and second, the classical ET group has a significantly higher percentage of aortic rupture, with more descending replacements and significant longer operation times accounting for higher mortality. In their conclusion they assume that the FET offers the benefit of a more ideal landings zone, although no evidence is provided. And yes, it may be more easy to put in a TEVAR in a FET, although also the pressurized conventional ET will stretch out (Figure 5) and in my experience it has never been impossible to complete it endovascularly. It is important that the length of the ET is at least $10 \mathrm{~cm}$, first to allow grasping of the floppy ET in open thoracoabdominal surgery but also with 4-6 large clips distally, combined with radiopaque dots on the prosthesis, as to facilitate adequate TEVAR positioning.

And what about the possible benefit of the zone 2 implantation of the Frozen. Let's look at a study from Bologna (12). One can read that this is a complex group of patients including Type A dissection and in half of the cases reoperation. Within the zone 2 group, the latest era, the separate graft technique was predominantly used with the Thoraflex prothesis. And yes, recurrent nerve palsy did go down although not significant, but mortality is the same for both groups over time and in total still high comparing to our series of the classical ET as investigated by our residents from Bologna as mentioned above (4). Paraplegia has gone down, however, also the rate of dialysis and abdominal complications reflecting more the change to the separate graft technique, with significantly shorter body ischemic time. 


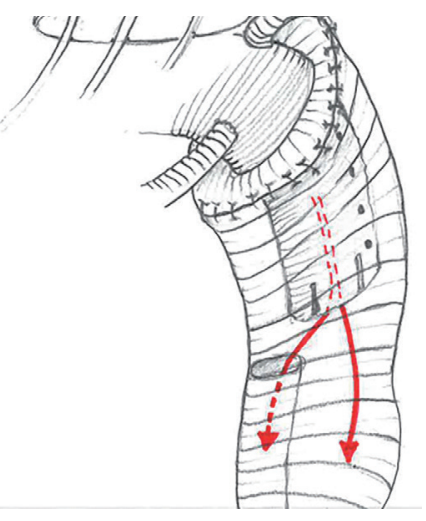

Figure 6 Cut out the intimal web as far as possible to allow both true and false lumen perfusion, to prevent thrombosis and to prevent paraplegia.

Arch surgery for chronic dissection is mostly elective and mortality should be low. This could be seen for the extensive experience from Hannover (9). In chronic dissection arch surgery first the false and true lumen are sutured together and then the FET is inserted in the true lumen and fixed. I was trained in the Antonius Hospital in Nieuwegein, the Netherlands, to always cut out the web as far as possible, to allow a both lumen perfusion, to prevént thrombosis and to prévent paraplegia; I have always done since (Figure 6).

Only by adding spinal cord drainage and diminishing the lengths of the FET, spinal cord injury did go down. What is puzzling to me is why the paraplegia rate for degenerative aneurysm surgery is still of concern (9) where false lumen thrombosis is not relevant. Also, puzzling is what the benefit will be for the long term? Leaving the FET short, and even shorter when implanted more proximal in zone 2, the expected benefit as for stopping the growth of the downstream aorta must be small.

What we do know is that reinterventions after FET are common if not inevitable (13) and what we begin to understand is the effect of the stiffness of an endoprothesis with regard to worsening heart function $(14,15)$ and with costs which are considerable. It is interesting to note that that de NICE draft Guideline in the UK suggested that patients with abdominal aortic aneurysms should not be offered endovascular repair if open surgical repair is feasible, although this draft is still under consultation. So, maybe the beginning of a reemphasis on the use of endografts in general? What we do know is that open post dissection surgery, also as second stage after classical

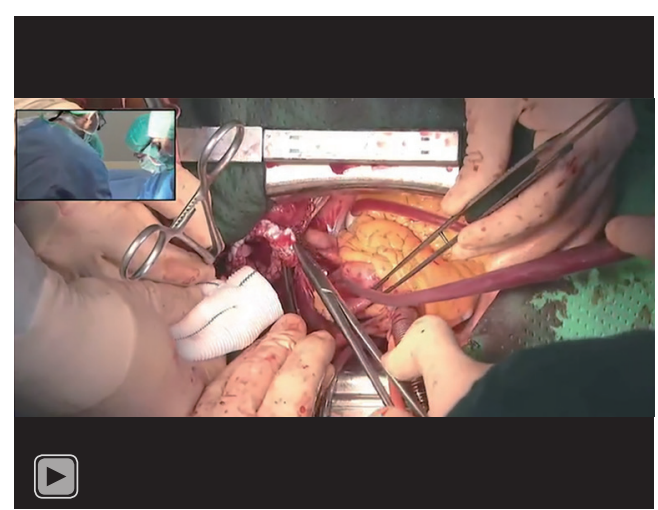

Video 1 Read at the Aortic Surgery Symposium. Bologna, November 2019.

ET, can be done with acceptable mortality and with a low paraplegia rate, with almost no need for reoperation at follow-up and with a life expectancy equal of that of the Dutch population (16).

Balancing short-term risk with higher mortality and higher stroke rates with possible long-term benefit is the key question; for me the balance is still in favor of a conventional ET. Thus far, I have usually found other ways to treat also localized aneurysms of the arch and or proximal descending aorta.

The text above is based on my lecture in the Aortic Surgery Symposium in Bologna in November 2019 where I was invited to address the question if there is still place for the classical elephant trunk in surgery of the arch and downstream aorta (Video 1). It is important to note that new results of the various techniques after this presentation have not been included in the text above.

In conclusion, the question should not be if the classical elephant trunk is still alive. It is alive and kicking and continuous to offers the golden standard. The question should better be if the innovative alternative already deserves the same status in aortic surgery. A question which has not been answered yet.

\section{Acknowledgments}

Funding: None.

\section{Footnote}

Provenance and Peer Review: This article was commissioned 
by the Guest Editors (Roberto Di Bartolomeo, Davide Pacini, Mohamad Bashir) for the series "The 10th Postgraduate Course on 'Surgery of the Thoracic Aorta' in Bologna" published in fournal of Visualized Surgery. The article has undergone external peer review.

Conflicts of Interest: The author has completed the ICMJE uniform disclosure form (available at https://jovs. amegroups.com/article/view/10.21037/jovs-20-7/coif). The series "The 10th Postgraduate Course on 'Surgery of the Thoracic Aorta' in Bologna" was commissioned by the editorial office without any funding or sponsorship. The author has no other conflicts of interest to declare.

Ethical Statement: The author is accountable for all aspects of the work in ensuring that questions related to the accuracy or integrity of any part of the work are appropriately investigated and resolved. All procedures performed in this study were in accordance with the Helsinki Declaration (as revised in 2013). The manuscript is waived from patient informed consent according to the ethics committee or institutional review board.

Open Access Statement: This is an Open Access article distributed in accordance with the Creative Commons Attribution-NonCommercial-NoDerivs 4.0 International License (CC BY-NC-ND 4.0), which permits the noncommercial replication and distribution of the article with the strict proviso that no changes or edits are made and the original work is properly cited (including links to both the formal publication through the relevant DOI and the license). See: https://creativecommons.org/ licenses/by-nc-nd/4.0/.

\section{References}

1. van Bakel TM, de Beaufort HW, Trimarchi S, et al. Status of branched endovascular aortic arch repair. Ann Cardiothorac Surg 2018;7:406-13.

2. Claassen L, van Workum F, Rosman C. Learning curve and postoperative outcomes of minimally invasive esophagectomy. J Thorac Dis 2019;11:S777-85.

3. Cefarelli M, Murana G, Surace GG, et al. Elective Aortic Arch Repair: Factors Influencing Neurologic Outcome in 791 Patients. Ann Thorac Surg 2017;104:2016-23.

4. Castrovinci S, Murana G, de Maat GE, et al. The classic elephant trunk technique for staged thoracic and thoracoabdominal aortic repair: long-term results. J Thorac Cardiovasc Surg 2015;149:416-22.

5. Sun LZ, Qi RD, Chang Q, et al. Is total arch replacement combined with stented elephant trunk implantation justified for patients with chronic Stanford type A aortic dissection? J Thorac Cardiovasc Surg 2009;138:892-6.

6. Di Eusanio M, Armaro A, Di Marco L, et al. Short- and midterm results after hybrid treatment of chronic aortic dissection with the frozen elephant trunk technique. Eur J Cardiothorac Surg 2011;40:875-80.

7. Pacini D, Tsagakis K, Jakob H, et al. The frozen elephant trunk for the treatment of chronic dissection of the thoracic aorta: a multicenter experience. Ann Thorac Surg 2011;92:1663-70; discussion 1670.

8. Jakob H, Tsagakis K, Pacini D, et al. The International E-vita Open Registry: data sets of 274 patients. J Cardiovasc Surg (Torino) 2011;52:717-23.

9. Shrestha M, Martens A, Kaufeld T, et al. Single-centre experience with the frozen elephant trunk technique in 251 patients over 15 years. Eur J Cardiothorac Surg 2017;52:858-66.

10. Shrestha M, Martens A, Krüger H, et al. Total aortic arch replacement with the elephant trunk technique: single-centre 30-year results. Eur J Cardiothorac Surg 2014;45:289-95; discussion 295-6.

11. Rustum S, Beckmann E, Wilhelmi M, et al. Is the frozen elephant trunk procedure superior to the conventional elephant trunk procedure for completion of the second stage? Eur J Cardiothorac Surg 2017;52:725-32.

12. Leone A, Di Marco L, Coppola G, et al. Open distal anastomosis in the frozen elephant trunk technique: initial experiences and preliminary results of arch zone 2 versus arch zone $3 \uparrow$. Eur J Cardiothorac Surg 2019;56:564-71.

13. Kreibich M, Berger T, Rylski B, et al. Aortic reinterventions after the frozen elephant trunk procedure. J Thorac Cardiovasc Surg 2020;159:392399.e1.

14. Hiraoka A, Toki M, Hayashida A, et al. Exercise stress echocardiogram for the evaluation of change in the ventricular-arterial interaction after thoracic endovascular aortic repair. Eur J Cardiothorac Surg 2019;55:632-8.

15. Yamashita Y, Oishi Y, Motomatsu Y, et al. Thoracic endografting increases cardiac afterload and leads to ventricular hypertrophy in dogs. Eur J Cardiothorac Surg 
2019;55:618-25.

16. Alfonsi J, Murana G, Smeenk HG, et al. Open surgical repair of post-dissection thoraco-abdominal aortic aneurysms: early and late outcomes of a single-centre study involving over 200 patients. Eur J Cardiothorac Surg 2018;54:382-8.

doi: 10.21037 /jovs-20-7

Cite this article as: Morshuis WJ. Classical Elephant Trunkold fashioned thus obsolete? J Vis Surg 2023;9:20. 